\title{
A Modering and Experimental Study on Accelerated Reliability Testing Method for Electric 4WD Vehicle
}

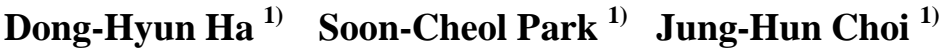 \\ 1) Hyndai motor Company, Durability Research Lab, \\ 150, Hyndai Yeinguso-ro, Namyang-eup, Hwaseong-si, Gyoenggi-do, 18280, Korea \\ (E-mail:dha@hyundai.com,testdrv@hyundai.com)
}

Received on July 6, 2018

\begin{abstract}
The e-4WD(electric four wheel drive) is the system that transfer powers to wheels and has an electric motor and reduction gear unit on a rear wheel axle. Durability test mode for e-4WD system is required. In this study, several types of sensors were equipped on the electric vehicle which has e-4WD system to measure not only vehicle behaviors and driving torques, but also driving condition. As a result of the analysis on vehicle behaviors in each driving condition, the e-4WD module operating durability mode which is optimized for new hybrid plaform's dimension has been developed. System vibrational test method and revolutional test method for motor and reduction gear unit have also been developed. Moreover, the modeling that is available for developing accelerated durability test mode with only e-4WD Design parameters and driving condition has been studied.
\end{abstract}

KEY WORDS: EV and HV systems, motor drive system, standardization, e-4WD Test Methodology, Electric 4WD Vehicle [A3]

\section{Introduction}

e-4WD is consists of Electric motor, reduction gear unit and battery that are equppied in rear wheel axle. It transfers power to each wheels and makes $2 \mathrm{WD}$ into $4 \mathrm{WD}$ if e-4WD is installed. e4WD system tries to maintain existing platform and it's easy to be part of internal combustion without any complicity. Therefore, not only is it 4WD technology easily available, but it also has increased fuel-efficiency technology. Moreover, driving performance can be enhanced with assist of a rear motor when initial acceleration or driving uphill road. On city roads, driving with only motor power is possible and on rough or slippery roads, 4WD driving is also available. In order to adapt final e-4WD prototype for the first time, developing test procedure is necessary to verify durability performance. Figure 1 is the configuration of e-4WD system. In this study, vehicle behaviors, driving torque and driving condition are measured with instrumented hybrid electric vehicle to develop optimized module operating test procedure. Moreover, the development of test modes for system vibration durability and reduction gear unit are studied. Lastly, modeling of durability test mode only with design parameters and driving condition is studied.

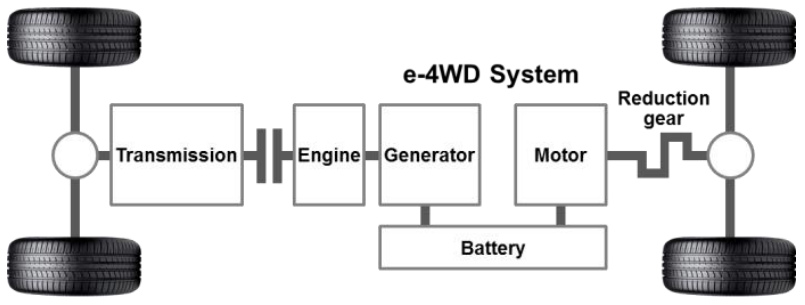

Fig. 1 Schematic of the powertrain configuration of e-4WD hybrid system

\section{A study on Accelerated Reliability Testing Method}

2.1. The development of operating durability test mode for e-4WD hybrid system

First, the motor torque and revolutions of the vehicle are measured. Test condition for each driving modes with customer usages applied is established. Motor usage distribution for each e4WD modes equivalent to field conditions is calculated to build the accelerated test modes. Also, RMH(rotating moment histograms) method is used to calculated damages on system which uses T-N curves. Torques and revolutions are converted with RMH method. Event cycles of the test mode which is calculated by contact damages on bearing parts are determined. Therefore, the test mode can be established ${ }^{(1)}$. Driving mode consists of 4 types which are pure EV mode, Auto mode (optimized fuel consumption), SPORT mode(higher engine RPM, faster gear shift), Forced 4WD mode (driven with engine and motor). Different types driving condition such as proving grounds testing, various events on field and different driving modes on the same courses and so on are measured. Vehicle motor torque, the number of revolutions, drive shaft torque, wheel force, moment and CAN are measured. In addition to this proportion, which driving modes are used or how long the certain mode is used should be also considered. Therefore, the proportion of e-4WD usages of customers considered accoding to EV mode, AUTO mode, SPORT mode and 4WD mode.

Next procedure is fatigue damage analysis method to develop rotating e-4WD module operating durability mode. When calculating damages on Powertrain especially with a lot of rotating parts, RMH method is used. Figure 2 shows the relationship between torque and number of revolution of rotating parts. The number of revolution $\left(n_{i}\right)$ is calculated by fixed torque level $\left(T_{i}\right)$ during discrete time intervals $\left(\Delta t_{i}, i=1,2, \ldots, \mathrm{m}\right)$ in 
revolution speed time history $(r p m(t))$. The number of revolutions at $\mathrm{Ti}$ can be expressed as follows:

$$
n_{i}=\sum_{i=1}^{m} \int_{\Delta t_{i}} r p m(t) d t
$$

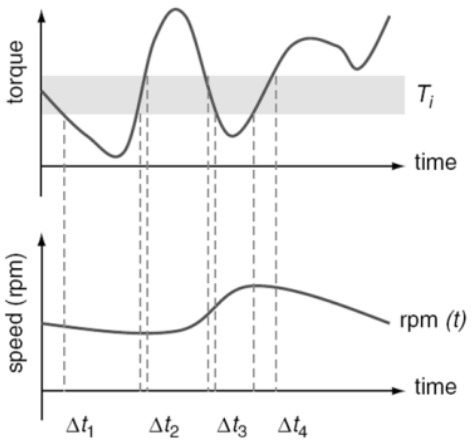

Fig. 2 The relationship between torque and number of revolution of rotating parts

As you can see Figure 3, fatigue test build fatigue resistance curve T-N(Torque-Life Curve) to measure fatigue life on driving system using drive shaft with various torque levels. Linear damage method is used for calculating cumulative damages on shaft which uses T-N curve and rotating moment histograms from proving grounds testing and it's described as formula (2). Where $\mathrm{D}$ denotes the summation of each damage(di) which is the proportion of calculated number of revolution in torque level $T_{i}$ and fatigue life $N_{i}$ equivalent to the torque level calculated from T-N curve ${ }^{(2)}$

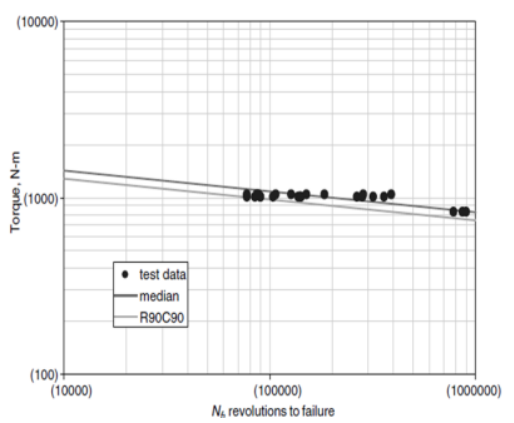

Fig. 3 Fatigue test data of a differential gear set

$$
D=\sum D_{i}=\sum \frac{n_{i}}{N_{i}} \geq 1.0
$$

In order to develop e-4WD module operating durability test method, the distribution of motor torque and number of revolution equivalent to field conditions considering customer usages and driving condition is analyzed in Figure 4. In field, motor torque and the number of revolution are used evenly in all area. e-4WD system is operated with rear motor and battery in low-speed driving. However, when driving fast or acceleration or uphill driving, hybrid engine and motor operate together to increase driving power. When driving downhill, regenerative energy is stored and charged in battery. The operation overview of e-4WD Hybrid system is shown in Figure 5.

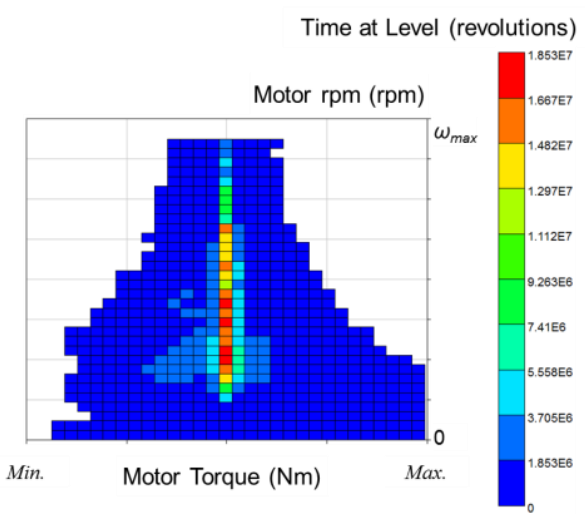

Fig. 4 The distribution of motor torque and number of revolution

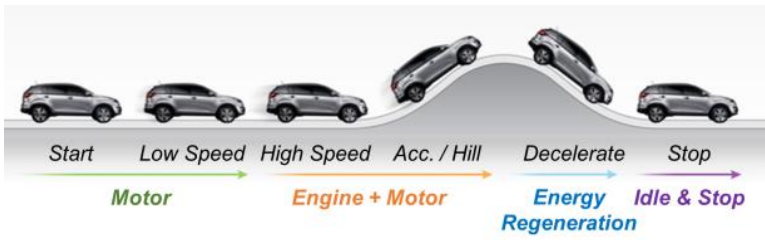

Fig. 5 The operation overview of e-4WD hybrid system

Acceleration factors that accelerate e-4WD system need to be studied to develop shortened durability mode with various events, motor usages, usage distribution in field. Analysis of motor usages distribution according to a number of condition such as go and stop, high-speed acceleration, deceleration and steady speed, regenerative braking, mid-speed acceleration, deceleration, up hill and down, slip is shown in Figure 6. Representative acceleration factors for four different driving patterns are shown. Representative battery discharging acceleration factor and charging acceleration factor by regenerative energy can be calculated using the number of revolution of motor $\omega$, maximum number of revolution of motor $\omega_{\max }$.

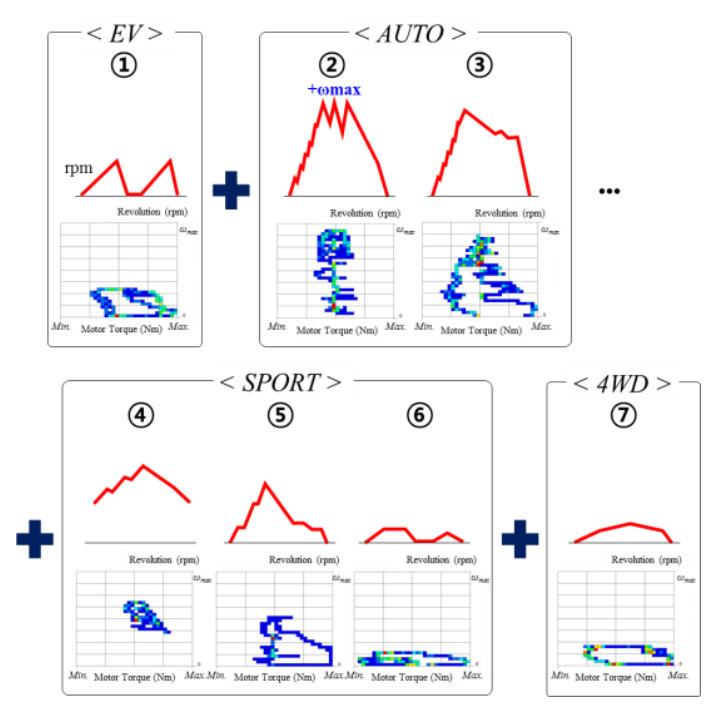

Fig. 6 Results of acceleration factor analysis according to operating modes of e-4WD system 
Operating durability test profile considering the operating condition of motor, field usage distribution and damages on e4WD system and parts is created. Figure 7 shows the upgraded result of motor usage distribution in field with the combination of acceleration factors and the number of different cycles for each modes. It needs low speed if battery is discharged for motor power. However, the area that needs high torque is replaced with city EV mode (1) which starts after stopping such as Go\&Stop. Charging mode of medium/high torque area can be replaced with AUTO mode. Low charging and discharging area of battery with high RPM distribution can be accelerated by AUTO mode (2) and steady speed/regenerative condition mode (3). Discharging mode in medium-high torque area is equivalent to the SPORT mode with medium speed and acceleration and deceleration (4). High torque and low/medium RPM area can be added by SPORT mode with low-speed and acceleration and deceleration (5). The charging mode with low-speed and high torque can be simulated by regenerative mode and SPORT up hill and down or 4WD slip mode. The newly developed accelerated durability test mode that is equivalent to field conditions is shown in Figure 8.

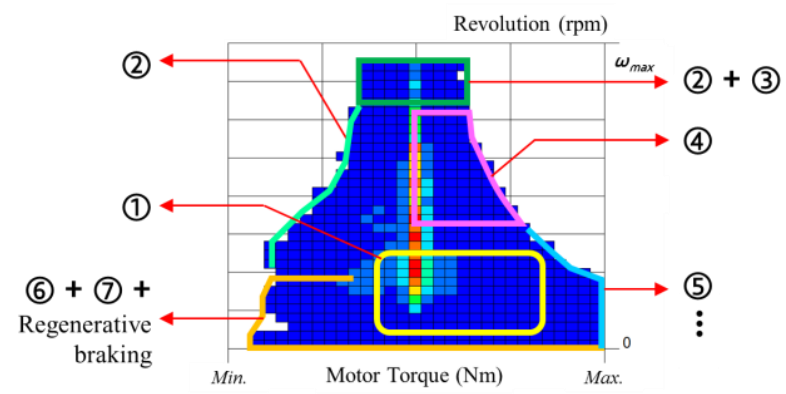

Fig. 7 The upgraded result of motor usage distribution in field with the combination of acceleration factors and the number of different cycles for each modes

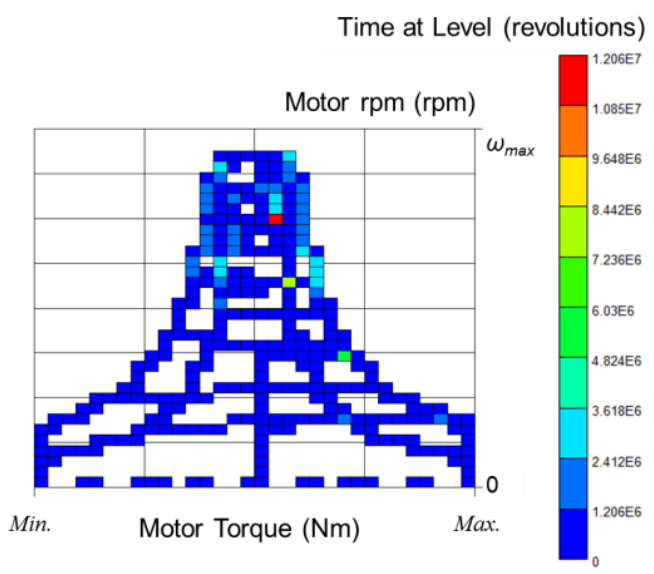

Fig. 8 The newly developed accelerated durability test mode

2.2.The development of vibration durability test procedure for e-4WD hybrid system

Vibration durability is also as important as operating durability to validate new durability mode of e-4WD system. Figure 9 shows dynamic modeling of mission synthesis method. The test signal is usually generated as a PSD of random loading to be applied over a period of time. It represents natural frequency output in single degree of freedom of a model and fatigue damage of FDS(fatigue damage spectrum). Shock response spectrum refers to the relation between maximum load amplitude of SDOF(single degree of freedom) and natural frequency of the system ${ }^{(3)}$. The fatigue damage of the total life time is calculated as the summation of fatigue damage spectrum for vibration during the period of the e-4WD system used. FDS for natural frequency can be expressed as follows:

$$
F D S\left(f_{o}\right)=f_{o} \cdot T \cdot \frac{K^{b}}{C} \cdot\left[\frac{Q \cdot G_{\ddot{x}}\left(f_{o}\right)}{2\left(2 \pi \cdot f_{o}\right)^{3}}\right]^{b / 2} \cdot \Gamma\left(1+\frac{b}{2}\right)
$$

$\Gamma$ is duration over which PSD persisted, $K$ is spring stiffness. $b$ and $C$ is basquin's SN exponent and coefficient. $Q$ is dynamic amplification factor. $\mathrm{G}_{\ddot{x}}\left(f_{0}\right)$ is the value of the acceleration input PSD expressed in $\mathrm{Hz}$ at natural frequency fo. The newly developed random vibration durability test mode that is equivalent to field conditions is shown in Figure 10. The synthesized random PSD profile equation is derived as shown in the following equation (4). Teq is desired test duration, $G_{0}\left(f_{0}\right)$ is the synthesized vibration PSD profile. $K$ is product of safety factor and test factor.

$$
G_{o}\left(f_{o}\right)=\frac{2\left(2 \pi \cdot f_{o}\right)^{3}}{Q} \cdot\left[\frac{k \cdot \sum F D S\left(f_{o}\right) \cdot C}{k^{b} \cdot f_{o} \cdot T_{e q} \cdot \Gamma\left(1+\frac{b}{2}\right)}\right]^{b / 2}
$$

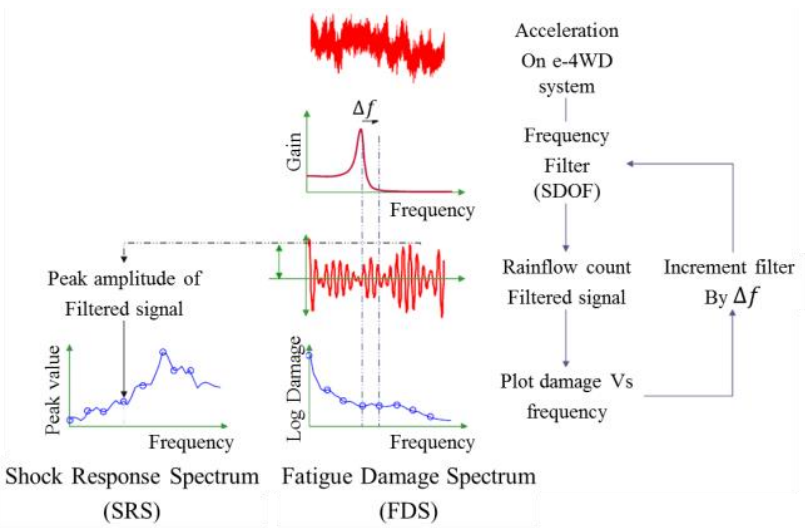

Fig. 9 Schematic flowchart illustrating the SRS and FDS calculation process

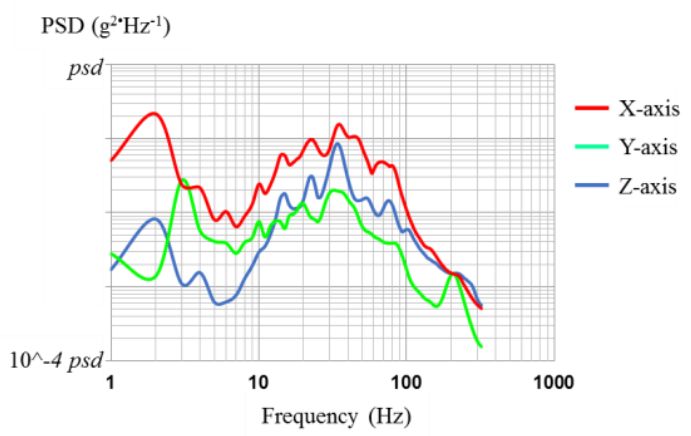

Fig. 10 Random vibration profile for e-4WD system

It is necessary to test not only the anti-vibration but also the vibration caused by the impact. The impact SRS(shock response 
spectrum) is used to determine the peak amplitude of this load during the impact vibration event of e-4WD system. A vibration evaluation test is required in consideration of acceleration amplitude and frequency. A test method that takes into account not only general vibration but also vibration due to impact is needed. SRS is used to determine the peak amplitude of this load during the impact vibration event of e-4WD hybrid system. The vibration evaluation test is required in consideration of acceleration amplitude and frequency. Figure 11 shows the process of setting the impact vibration test method for the e-4WD module. Each impact event is set to a representative SRS using the envelope function as the final shock specification, test time $(m s)$ and impact vibration $(g)$
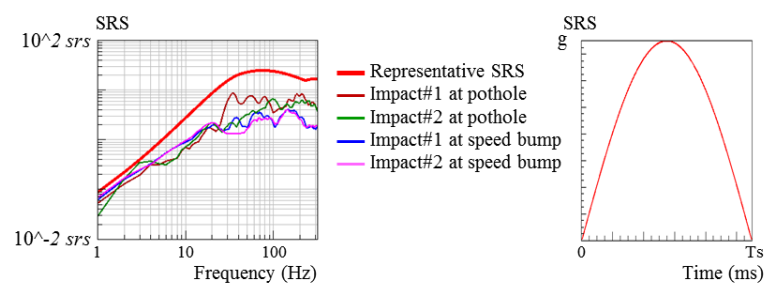

Fig. 11 Shock vibration profile for e-4WD system

2.3. A modeling study on accelerated reliability testing method for e-4WD hybrid system

The basic components of the e-4WD system are motor, battery, and reducer. Of these, the mechanically operating motor and the speed reducer are determined by the number of revolutions of the motor and the torque specification. According to the change of e4WD design specification in the future, it takes a lot of manpower and time to proceed with driving condition such as proving grounds testing and measurement, the field driving condition, the user selection mode, and the test mode setting. Therefore, it is necessary to study the test method through the modeling of the test based on the design specification. The modeling methodology was studied to make it possible to simulate by mechanical stage and design specification without measuring by actual vehicle. Figure 12 shows the outline of modeling for e-4WD system.

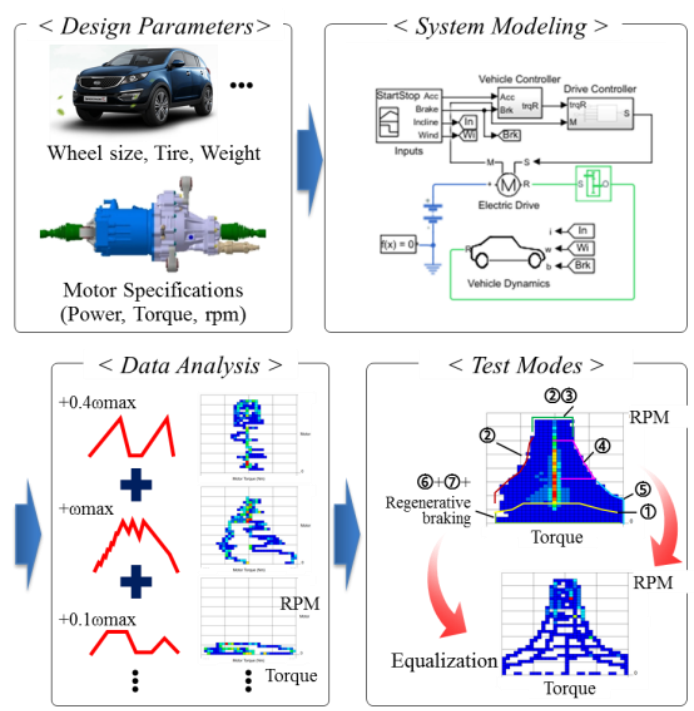

Fig. 12 The methodology of modeling for e-4WD system

\section{Conclusion}

The results of a modeling and experimental study on accelerated reliability testing method for electric $4 \mathrm{WD}$ vehicle are as follows.

Firstly, by measuring e-4WD module characteristics and field conditions and by analyzing e-4WD module acceleration factor considering e-4WD usage distribution, we deleoped the e-4WD module operating durability test modes which is optimized for new hybrid plaform's dimension has been developed.

Secondly, this paper described the methodology for the general random and impact vibration for e-4WD hybrid system. The vibration durability test affected the noise of the motor internal bearing of the e-4WD module and the damage of gear teeth of the motor reducer. Therefore the vibrations were amplified at the e4WD system, rotating parts such as bearings and gears generated noise.

Thirdly, through the modeling of the test based on the design specification, the modeling methodology was studied to make it possible to simulate by mechanical stage and design specification without measuring by actual vehicle.

Finally, based on the modeling and results of the e-4WD durability test method developed in this study, the methodology of modeling for e-4WD system that enables preliminary verification of the actual vehicle for the parts dedicated to environmental vehicles and rotating parts is suggested.

This paper is written based on a proceeding presented at JSAE 2018 Annual Congress.

\section{References}

(1) Ha, D., Park, S., and Shin, C. : Development of Accelerated Reliability Testing Method for Electric Vehicle Motor and Battery System, SAE Technical Paper 2014-01-0748, doi:10.4271/2014-01-0748 (2014).

(2) Yung-Li Lee, Jwo Pan, Richard Hathaway, Mark Barkey : Fatigue Testing and Analysis, Elsevier, pp. 313-336 (2005).

(3) A. Halfpenny : Accelerated Vibration Testing Based on Fatigue Damage Spectra, Proceeding of Aerospace Testing Expo, Hamburg, Germany, April 4-6 (2006).

(4) Kim, N., Rask, E., and Rousseau, A. : Control Analysis under Different Driving Conditions for Peugeot 3008 Hybrid 4, SAE Int. J. Alt. Power, Vol.3, No.1, doi:10.4271/2014-01-1818 (2014).

(5) D. W. Gao, C. Mi, A. Emadi : Modeling and Simulation of Electric and Hybrid Vehicles, Proceedings of the IEEE, Vol.95, No.4, pp. 729-745, doi:10.1109/JPROC.2006.890127 (2007).

(6) Lin, C.C, Filipi, Z., Wang, Y., et al. : Integrated, FeedForward Hybrid Electric Vehicle Simulation in SIMULINK and its Use for Power Management Studies, SAE Technical Paper 2001-01-1334, doi:10.4271/2001-01-1334 (2001). 